\title{
EUROSOIL 2012 bioremediation session: preface to special issue
}

\author{
Edoardo Puglisi • Marco Trevisan • \\ Tim Grotenhuis · Lorenz Adrian
}

Published online: 26 May 2013

(C) Springer Science+Business Media Dordrecht 2013

The fourth International Conference of the European Confederations of Soil Science Societies (ECSSS) took place in Bari, Italy from the 2nd to the 6th of July 2012. The general theme of the Conference was "Soil science for the benefit of mankind and environment", and has been structured in 13 general topics that included 63 symposia and 5 workshops, as well as 16 special events including round tables, open debates and short course. As a whole, the Scientific Programme included more than 2,300 presentations, divided in more than 660 orals and more than 1,600 posters.

A bottom-up approach was applied by the Conference organizing committee for the development of the scientific sessions: conveners and co-conveners thus proposed scientific themes and circulated calls for abstracts. Our aim was to promote a scientific

E. Puglisi $(\bowtie)$

Istituto di Microbiologia, Università Cattolica del Sacro

Cuore, Via Emilia Parmense 84, 29122 Piacenza, Italy

e-mail: edoardo.puglisi@unicatt.it

\section{Trevisan}

Istituto di Chimica Agrariaed Ambientale, Università

Cattolica del Sacro Cuore, Via Emilia Parmense 84,

29122 Piacenza, Italy

e-mail: marco.trevisan@unicatt.it

T. Grotenhuis

Sub-Department of Environmental Technology,

Wageningen University, P.O. Box 17,

6700 AA Wageningen, The Netherlands

e-mail: tim.grotenhuis@wur.nl symposium where the issue of bioremediation of polluted terrestrial environments could be addressed by a multidisciplinary approach taking into account both microbiological and chemical issues. We thus initiated a session entitled "Bioremediation of soils and sediments contaminated with organic chemicals: assessing and overcoming chemical and microbiological constraints", with the aim of identifying the main constraints limiting the success of bioremediation practices and to show how scientific approaches on the interface between chemistry, microbiology and engineering sciences can help overcoming them. The session included (i) multi-disciplinary studies linking chemical assessment of pollutant bioavailability with biological measurement of the presence and activity of pollutant-transforming organisms; (ii) high-throughput

\author{
T. Grotenhuis \\ Bornse Weilanden 9, 6703 HD Wageningen, \\ The Netherlands \\ L. Adrian \\ Department Isotope Biogeochemistry, \\ Helmholtz Centre for Environmental Research-UFZ, \\ 04318 Leipzig, Germany \\ e-mail: lorenz.adrian@ufz.de
}


analyses of pollutant-degrading microbial consortia in aerobic and anaerobic environments; (iii) studies investigating the effects of soil properties on organic contaminant bioavailability; (iv) methods and approaches to enhance pollutant availability and degradation; and (v) research showing the successful application of specific bioremediation strategies.

To the call 56 abstracts were submitted, 33 as suggested oral and 23 as poster contribution. Abstracts came from all over the world including Italy (12 abstracts), Germany (7) France (5), Brazil, Iran, Hungary, Serbia, The Netherlands, Tunisia, United States of America (2 each) and from Albania, Australia, Azerbaijan, Cameroon, Canada, Chile, China, Croatia, Egypt, India, Latvia, Martinique, Mexico, Portugal, Romania and Spain (one abstract each). The range of topics covered was also vast. Organic pollutants were mostly studied, with 39 abstracts, followed by inorganics (9) and mixed pollutions (8). Among organics, polycyclic aromatic hydrocarbons were mostly represented (12 abstracts), followed by petroleum hydrocarbons (6), and endocrine disruptors (3). Other class of molecules addressed in at least one study were BTEX, chlordecone, isoproturon, nitroaromatics, olive mill wastes, organophosphates, pesticide mixtures, phenols and volatile hydrocarbons, showing the wide spectrum of topics of actual research. Studies on inorganics generally focused on mixtures, with the exception of one study focusing on lead and one on uranium. Laboratory studies predominated the lot (28 abstracts), followed by field studies (20) and greenhouse (1 study). Six studies can also be classified as theoretical, since they were not lab or field study but are based on modelling or on the proposal of new approaches for the bioremediation of polluted sites. Finally, soil was the mostly studied environment (39 abstracts), followed by sediments (9). Glaciers and groundwater were also represented by two studies.

From these submitted abstracts, a difficult choice was made to select 14 of them, which were presented as orals during the afternoon of Friday 6th of July. A call for submission of scientific papers to be published in the present special issue was launched during the call for abstracts, and closed 1 month after the symposium. Twelve original manuscripts were submitted, and after peer-review ten were finally published and are here presented. In the first one, "The glutathione S-transferase gene superfamily: an in silico approach to study the post translational regulation" and in silico bioinformatics study was carried out in order to assess the diversity of glutathione-S-transferase, an important gene in phytoremediation. The two following papers, "Potential of kenaf (Hibiscus cannabinus) and corn (Zea mays) for phytoremediation of dredging sludge contaminated by trace metals" and "A real-scale soil phytoremediation" report field studies were phytoremediation was employed with promising results; in the first case to remediate zinc and cadmium pollution by using kenaf and corn plants, in the second target pollutants were mixtures of heavy metals and hydrocarbons, and the plant used belonged to the genera Populus, Paulownia and Cytisus. In the paper "Decontamination and functional reclamation of dredged brackish sediments", it is shown that the phytoremediation of mixtures of organic and inorganic pollutants by different plant species can be improved by a bioaugmentation approach based on the addition of soil and compost to contaminated sediments. In the paper, "Decontamination of a polychlorinated biphenylscontaminated soil by phytoremediation-assisted bioaugmentation", it is shown that the fescue plants can improve the biodegradation of PCBs making use of the indigenous microbial community, while the inoculation of a well-studied PCB degrader (Bulkholderia xenovorans LB400), did not significantly increase degradation activity, albeit the survival of the strain was proven by molecular quantifications. Transformation of volatile chlorinated hydrocarbons was assessed and modelled in laboratory conditions in the contribution "(Bio-)remediation of VCHC contaminants in a Technosol under unsaturated conditions", where it is shown that pump-and-treat systems can be improved by the injection of microemulsions of Dehalococcoides strains. The use of microorganisms in bioremediation was also the focus of the following papers. The paper "Enrichment of hexachlorobenzene and 1,3, 5-trichlorobenzene transforming bacteria from sediments in Germany and Vietnam" reports the enrichment of dechlorinating bacteria from German and Vietnam sediments catalysing new pathways of chlorobenzenes dechlorination and avoiding persistent end products. The manuscript "Inoculation of PAHdegrading strains of Fusariumsolani and Arthrobacteroxydans in rhizospheric sand and soil microcosms: microbial interactions and PAH dissipation" show interesting interactions between the fungus and the bacterium as model strains for the dissipation of PAH mixtures. Experiments were carried out by inoculating 
these strains into non-sterilized soils, and thus provide insights on the behaviour of degrading strains inoculated in the presence of complex microbial communities, which is one of the main constraints of bioremediation activities. The effects of aging and bioavailability on the bioremediation of petroleum hydrocarbons was assessed in the paper "Biodegradation of aged diesel in diverse soil matrixes: impact of environmental conditions and bioavailability on microbial remediation capacity": as a very interesting outcome of the study, the effect of matrix types on the processes was also considered, as well as correlations between microbial monoxygenase genes abundance and bioremediation extent. Natural attenuation was studied in the paper "Long-term assessment of natural attenuation: statistical approach on soils with aged PAH contamination", using PAHs as model molecules: the results here presented show good efficiencies of this approach, which present several advantages as compared to more costly approaches.
The symposia we hosted in EUROSOIL 2012 and the papers here presented prove that the bioremediation of soils and sediments is still a popular and relevant topic, stimulating a vast number of important research. We were impressed by the variety of approaches presented, and by the fact that basic ecological issues such as the interaction of inoculated strains in soils or sediments with the indigenous microflora are important determinants of the success of bioremediation practices. Other important outcomes are the comparisons of methods applied, ranging from chemical measurements to assessments by molecular techniques, and the importance of coupling different approaches (e.g., phytoremediation and bioaugmentation; bioavailability assessments and quantification of microbial degradation genes) as a key factor for the success of bioremediation practice. We generally believe that the papers presented in this special issue provide a representation of the highquality research being carried out around the World in the bioremediation of polluted soils and sediments. 